\title{
OBSTACLE DETECTION AND AVOIDANCE FOR AUTONOMOUS ELECTRIC VEHICLE USING ARDUINO
}

\author{
Miss. Shusmita M. Rathod \\ Student, Electronic Department \\ Sanjay Ghodawat University, Kolhapur, \\ Maharashtra, India
}

\author{
Mrs. S. K. Apte \\ Prof., Electronic Department \\ Sanjay Ghodawat University, Kolhapur, \\ Maharashtra, India
}

\begin{abstract}
Now a days Autonomous electric vehicle are the current research trends in the automobile industry and future vehicle used by humans. However, the commercial realization of autonomous vehicles remains a significant challenge. My project put forward an obstacle avoider robotics vehicle that uses and sharp distance IR sensor for this purpose the system uses an ATmega2560 Arduino microcontroller to achieve this functionality. The first part of my project is to design a system in such a way that it avoids any kind of obstacle that comes in its path. The vehicle achieves this functionality with the help of sharp distance IR sensors, ATmega2560 microcontroller, and motors. The system allows the vehicle to guide itself during obstacle occurred. The obstacle detection is done using Sharp distance IR sensors. After detecting the obstacle and this signal is passed to the ATmega2560 microcontroller on receiving the signals it guides the vehicle to moves in a different direction by actuating the motors through the motor driver.
\end{abstract}

Keywords-Autonomous Vehicle, Obstacle Detection, Obstacle Avoidance, Sharp Distance IR sensors long range $(20 \mathrm{~cm}-150 \mathrm{~cm})$ and short range $(10 \mathrm{~cm}-80 \mathrm{~cm})$, ATmega2560 Arduino microcontroller, Dual shaft geared DC motor, 1298n dual h bridge steeper motor controller.

\section{INTRODUCTION}

An autonomous vehicle navigates to the destination without the help of a human driver. The main aim of an autonomous vehicle is to increase the safety and avoid the error occurred during human driving. An autonomous car can be controlled by using the motor driver and a combination of various sensors such as ultrasonic sensor, infrared sensors, radar, Lidar, camera, etc. autonomous vehicle is mainly designed for safety purpose because the problem of an accident occurred Because of humans such as distraction on driving because of cell phones.
The autonomous vehicles consist of many advantages such as fuel economy, the numbers of crashes are reduced, and various types of pollution are also decreased. The driving of an autonomous vehicle is safe even though the driver is tired. The Autonomous vehicle is safe as compared to human driver almost in all cases. Also, there are some disadvantages of autonomous vehicle suppose the car has no driver if the crashes occurred without driver then whose fault exactly the software designer fault or the owner fault, in that case, there are lots of problems get arise for financial responsibility. Another disadvantage such as reading road signs is very difficult for a robot to suppose the GPS and other technologies are not registered for an obstacle or recent changes in road condition and newly posted signs. Sometimes the sensor gets failed because of weather condition this may not work during heavy snowfall. Human behaviour such as hand signals is very difficult for a computer to recognize.

These self-driving vehicles integrate on US roadway by using these five levels of driver assistance technology in coming years. According to the scope of automation, levels 0 to 5 are defined. With level 0 has no automation while level 5 signifying full automation in all conditions. All these levels are defined by standards organization Society of Automotive Engineers (SAE) according to automated driving systems. In Level 0 Driver has complete control of the vehicle at all times. Some vehicle controls are automated in Level 1 e.g. automatic braking. In Level 2 Two or more controls can be automated at the same time, e.g. cruise control and lane keeping. The Level 3 driver can provide control in certain circumstances. Driver not expected to play any part in the driving process at level 4. And the last level is Level 5 in that level the vehicle performs autonomously in every situation, including unusual and extreme conditions.

An electric vehicle also referred to as an electric drive vehicle, is a vehicle which uses one or more electric motors for moving the car in any direction. To power, the battery in the electric 


\section{International Journal of Engineering Applied Sciences and Technology, 2019 \\ Vol. 4, Issue 1, ISSN No. 2455-2143, Pages 120-125 \\ Published Online May 2019 in IJEAST (http://www.ijeast.com)}

vehicle the electricity is the main fuel used. The battery is the energy storage device used in the EV.

For design automated driving functionality automotive companies use MATLAB and Simulink software. MATLAB provides an accurate solution for the user problem and produces accurate code. The result can be easily obtained using MATLAB software rather than using expensive software for programming. Some functionality of Simulink is the same as MATLAB. Simulink uses block by simply clicking and dragging the block into the model window the system can be developed.Instead of writing code for a particular problem. Most of the companies use MATLAB and Simulink software because Simulink software reduces the cost of the design and also compact the size of the design. And the time required for design a system is also reduced.

\section{HARDWARE AND SOFTWARE COMPONENTS}

\section{HARDWARE}

\section{A. ARDUINO Mega Board}

Arduino is microcontroller it is part of a computer that runs one program again and again. Arduino board uses a variety of microprocessors and controllers. The boards consist of sets of digital and analog input/output (I/O) pins that may be interfaced to various boards or breadboards and other circuits. The boards feature Universal Serial Bus(USB), serial communications interfaces on some models, which are also used for loading programs from personal computers. The microcontrollers are typically programmed using the programming languages $\mathrm{C}$ and $\mathrm{C}++$. The integrated development environment (IDE) that are generally used to dump the project. The Arduino Mega 2560 is a microcontroller board based on the ATmega2560 8-bit AVR RISC based microcontroller. It consists of 54 digital input/output pins (of which 14 can be used as PWM outputs), 16 pins are analog inputs, 4 UARTs (hardware serial ports), a clock speed of $16 \mathrm{MHz}$ crystal oscillator, a USB connection, ICSP header a power jack, and a reset button. It simply started by connecting to a computer with a USB cable or applying $\mathrm{AC}$ or DC power using AC- to- DC adapter or battery. Arduino mega power pins are 5V, 3V, and GND. Pins are supplied less than $5 \mathrm{~V}$ voltage the board becomes unstable and if the supply voltage is more than $12 \mathrm{~V}$ there is a chance of damaging of voltage regulator because of overheat.

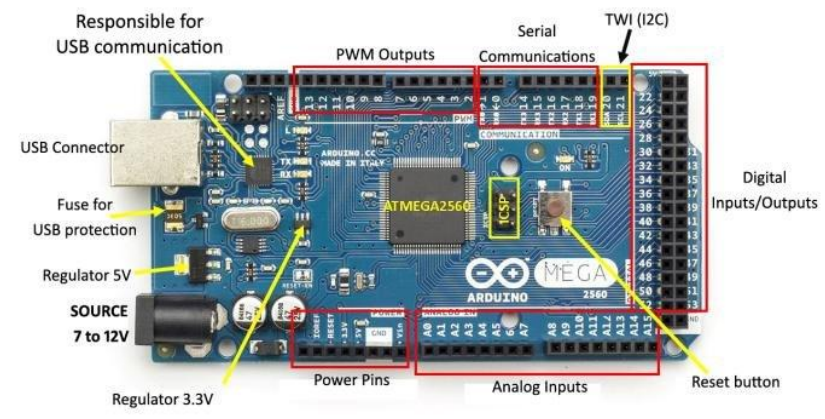

Fig.1. - Arduino Mega 2560

\section{B. L298n Dual H Bridge Stepper Motor Controller}

The L298N contains two H-Bridge circuits that can allow you to control the speed and direction of two DC motors, or control one bipolar stepper motor with ease. The L298N Hbridge module can be used with motors that have a voltage range between 5 and $35 \mathrm{~V} \mathrm{DC}$. If the supply voltage is up to $12 \mathrm{~V}$ you can also source $5 \mathrm{~V}$ from the board. The pins EnA and EnB of the motor controller L298 are used as the PWM pins. The DC motor run at a different speed and providing different high and low voltage levels to these pins of the motor control driver. It is widely used for robotic applications and well suited for connection to a microcontroller requiring just a couple of control lines per motor. It can also be interfaced with TTL logic gates, simple manual switches, relays, etc.

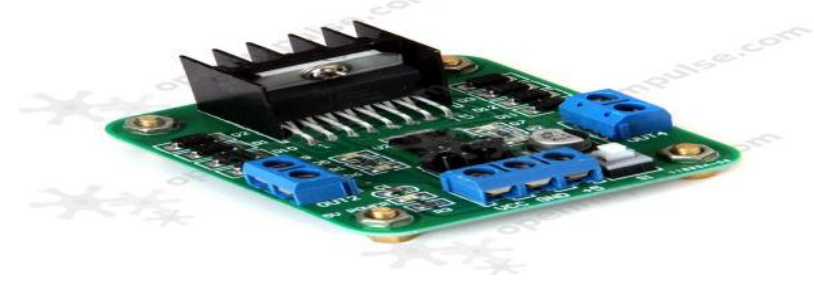

Fig.2. - L298n Dual H Bridge Steeper Motor Controller

\section{Dual Shaft De Geared Motor}

The electric energy can be converted into mechanical energy by using a DC motor. The rotation rate of a motor is proportional to the input voltage, and the rotation direction (clockwise or counterclockwise) depends on the polarity of the voltage (connecting the two wires of the motor to VCC and GND, or vice versa). The motors have a gearbox (reduction mechanism) with a 1:48 reduction rate, which means that for a complete rotation of the external shaft 48 rotations of the motor shaft are needed. Small DC motors are used in tools, toys, and appliances, etc. Larger DC motors are used in electric vehicles, compressor, refrigerators, etc. Electric wheel motor is ideal for robotics and model vehicle. It has a 1:48 gear ratio and max torque of $800 \mathrm{~g} / \mathrm{cm}$ at a minimum of $3 \mathrm{~V}$. 


\section{International Journal of Engineering Applied Sciences and Technology, 2019 \\ Vol. 4, Issue 1, ISSN No. 2455-2143, Pages 120-125 \\ Published Online May 2019 in IJEAST (http://www.ijeast.com)}

\section{Short Range IR Sensor}

GP2YOA21YKOF is a short range IR sensor generally used for measurement of distance which is consist of PSD(position sensitive detector), IRED( infrared emitting diode) and signal processing circuit, the environmental temperature because of the triangulation method the operating duration of short-range IR sensor is not easily changed. The short range IR sensor produced the output voltage corresponding to the distance detected by the sensor. The sensor operated on the supply voltage of $4.5 \mathrm{~V}$ to $5.5 \mathrm{~V}$. This sensor ranges from $10 \mathrm{~cm}$ to 80 $\mathrm{cm}$. the Current consumption capacity of short-range IR sensor is $30 \mathrm{~mA}$.

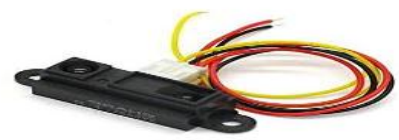

Fig. 4. - Short Range IR Sensor

\section{E. Long Range IR Sensor}

GP2YOA02YKOF is a Long range IR sensor generally used for measurement of distance which is consist of PSD(position sensitive detector), IRED( infrared emitting diode) and signal processing circuit, the environmental temperature because of the triangulation method the operating duration of short-range IR sensor is not easily changed. The short range IR sensor produced the output voltage corresponding to the distance detected by the sensor. The sensor operated on the supply voltage of $4.5 \mathrm{~V}$ to $5.5 \mathrm{~V}$. This sensor ranges of $20 \mathrm{~cm}$ to 150 $\mathrm{cm}$. the Current consumption capacity of short-range IR sensor is $33 \mathrm{~mA}$.

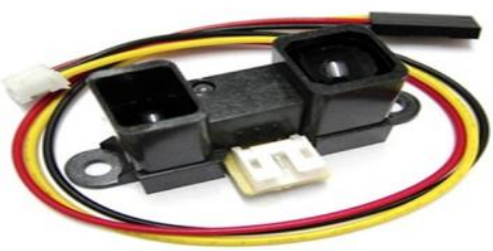

Fig. 5. - Long Range IR Sensor

\section{F. Servo Motor}

A servo motor is a rotatory actuator or linear actuator that allows precise control of angular speed, frequency, velocity, acceleration, angular position. Essentially it consists of an electric motor, feedback device, and electronic controller.

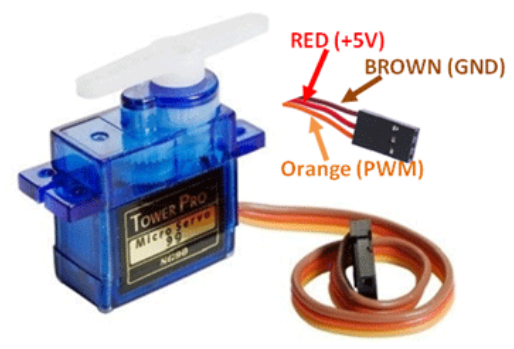

Fig.6. - Servo Motor

A servo motor is an electrical device which can push or rotate an object with great accuracy. Servo motor can be rotated from 0 to 180 degree, but it can go up to 210 degrees, depending on the manufacturing. Based on the Electrical Pulse of proper width that can be applied to its Control pin from that the degree of rotation of servo can be controlled. Servo motor that is generally used to rotate the object at some specific angles or distance. It is just made up of simple motor that can be run through a servo mechanism. If the motor is DC powered then it is called DC servo motor, and if it is AC powered motor then it is called an AC servo motor. The weights of a servo motor are generally measured in $\mathrm{kg} / \mathrm{cm}$.

\section{SOFTWARE}

\section{A. MATLAB}

MATLAB is developed by Maths Works. It allows matrix manipulations; plotting of data and functions; implementation of various types of algorithms; creation of user interfaces; also provide interfacing of programs written in other languages, such as $\mathrm{C}, \mathrm{C}++$, Java, and FORTRAN; it also performs some other function such as analysis of data; develop algorithms; and create models and applications. MATLAB consists of various built-in commands and math functions that can be used to perform some mathematical calculations, performing numerical methods and generating plots. MATLAB's has a power of Computational Mathematics. 


\section{International Journal of Engineering Applied Sciences and Technology, 2019 \\ Vol. 4, Issue 1, ISSN No. 2455-2143, Pages 120-125 \\ Published Online May 2019 in IJEAST (http://www.ijeast.com)}

\section{B. Simulink}

Simulink is a platform that has most of the functionality is the same as MATLAB but allows many engineers to design the system graphically and that can be interfaced with a block diagram. Basically, Simulink is a software package which is used for modeling, analyzing dynamical systems and simulating. It supports both linear and nonlinear systems, represented in continuous time, sampled time, or a hybrid of the two. Simulink provide a graphical environment in which you can model systems in the form of block diagrams, by simply clicking and dragging blocks into a model window. Simulink has a complete block library of sinks, sources, and subsystems (linear, nonlinear, and time-varying). It provides all the facilities of MATLAB when it running in Simulink. Using Simulink, the data displayed in scopes as the simulation stop. Outside the simulation environment, Simulink serves as the primary link for targeting to chips, boards, and cosimulation platforms by means of automatic code generation.

\section{LITERATURE REVIEW}

Adarsh, Kaleemuddin, Dinesh and Ramachandran (2016), explains about the performance comparisons of both ultrasonic sensor and infrared sensor. The performance comparison of IR and ultrasonic sensor usually based against various types of obstacles. The material used as obstacles such as Cardboard, Paper, Sponge, Wood, Plastic, Rubber and Tile. The parameter 'measured distance and actual distance in centimeter scale will provide the performance of sensor against the different type of material.

Sachin Modi (2002) author tells that the Obstacle avoidance is one of the critical issues in the designing of an autonomous vehicle. The obstacle avoidance is divided into two parts i.e. obstacle detection and avoidance control. In this paper the author explains about the different methods used for obstacle detection and avoidance such as fixed mounting of sonar sensor, rotating sonar sensor and laser scanner. The fixed mounted sonar sensors are present on the front side of the vehicle. The rotating sonar system consists of a Polaroid ultrasound transducer element mounted on a micromotor with encoder feedback. The motion of the motor is controlled using a Galil DMC 1000 motion control board. The data from the systems can be used to detect and avoid Obstacles.

Pavithra, Siva, Sharmila, Raja and Sushma(2017) describes the characteristics of different sensors such as ultrasonic sensor, infrared sensor, LASER which are used to avoid an obstacle in both indoor and outdoor environment. The disadvantages of the IR sensor are measured less distance as compared to LASER. Two Arduino boards required to connect LASER so for this reason the LASER used in less for obstacle detection. As compared to IR and LASER sensor ultrasonic sensor is more suitable for obstacle detection.
Jaycil and Randy (2015) they explains about the various technologies used for the development of autonomous vehicles and also explains the various advantages and disadvantages that are associated with different sensors used in autonomous vehicles. This paper also explained about the internal vehicle systems, external world sensing, sensors, systems, and various factors that play into autonomous vehicle design. Although the single sensor does not provide full information about the autonomous vehicle so that the fusion of sensor is used to drive the autonomous vehicle fully.

Domínguez, Onieva, Alonso, Villagra, and Gonzalez (2011) they explain about the development of car that can move without any driver. It just needs a command from the sense of the vehicle and provides a remarkable amount of information in the user, and it will start moving towards the destination. I have researched about my project, and I found some equipment that I need to build my vehicle. Some of them are LiDAR, Radar, GPS, and Sensors, etc. RADAR and Ultrasonic sensors will work as the range of $750 \mathrm{MB} / \mathrm{s}$, which is more than a human driver could even attain. I have referred the paper to learn more about LiDAR sensing. A LiDAR can process a detailed 3D image and 360-degree maps of the place.

Jie, Hui and Caijing (2009) they explain about the simulation tool are used for development of autonomous electric vehicle the author develops the simulation model of the autonomous vehicle based on MATLAB/Simulink software. It forms the vehicle sensor controller closed loop control system based on the relationship among autonomous vehicle, road conditions, and obstacles. The simulation model is used for control logic of Local Path Planning, and to design post-process of the raw data from sensors.

\section{EXPERIMENT SETUP AND PROCEDURE}

The experiment was conducted moving an autonomous robot which consists of sharp distance IR sensors, Arduino mega 2560 microcontroller, motor driver and DC motor. The model consists of the $12 \mathrm{~V} \mathrm{Li}$-ion rechargeable battery as the power source, DC motor and servo motor act as an actuator in which servo motor that is used for rotating the sensor from 0 degrees to 180 degrees. And DC motor used for power up the wheel so that it can rotate in specified directions. This obstacle detection can be carried out by managing the input and output signal coming from the IR sensor in that input and output signal management there consist of signal processing, signal conditioning, parameterization, and last part is integration. 


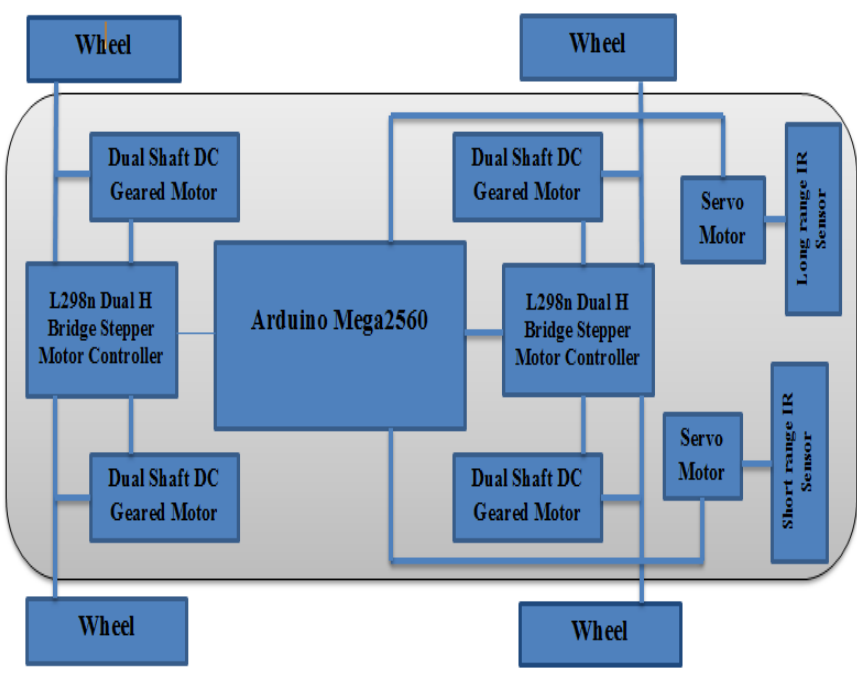

Fig.7. - Block Diagram of Experiment Setup

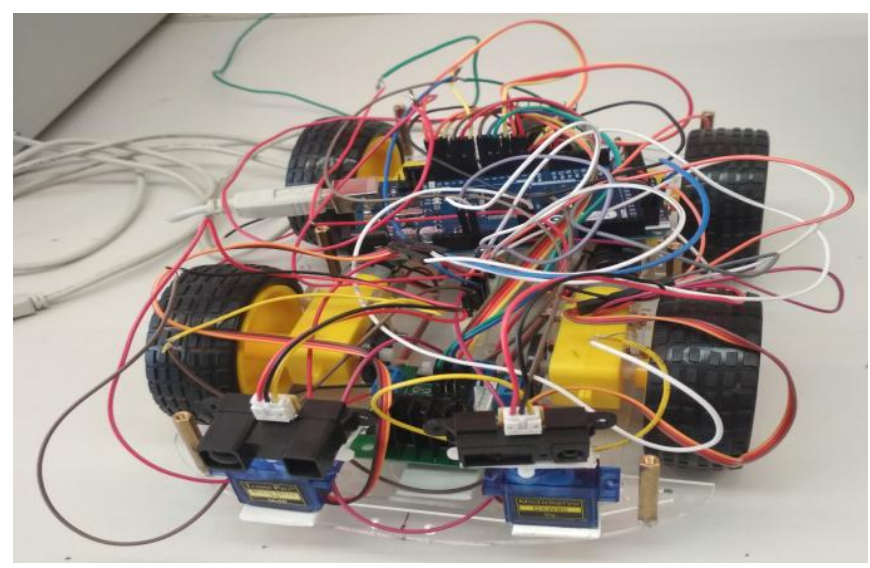

Fig.8. Photograph of Experiment Setup

Initially, both sharp distance IR sensors take the analog data from the analog pin of the Arduino board this data can be logged into the Arduino Mega microcontroller and Arduino mega microcontroller is connected to PC through USB. Sensors are mounted on the servo motor. And are placed on the front side of the vehicle. IR sensor starts rotating along with Servo when it is rotated from 0 degrees to 180 degrees. When the obstacle is detected both the IR sensor calculates the distance of obstacle by using the distance calculation formula such as the formula used for the short range is

Distance $(\mathrm{cm})=4800 /($ Sensor Value -20$)$
Servo motor calculates the obstacle angle through the starting and end point of an obstacle. After calculation of the distance from IR sensor which is out of IR sensor that can be given to the input of motor controller according to distance the condition is applied in the state flow such as for particular distance motor rotates in particular direction by keeping speed constant and varying distance and time. The complete system is developed in Simulink environment by using the state flow and conditions are applied in a State flow. The last part of my project is the integration of both the system that is obstacle detection using both sharp distance IR sensor and motor controller. The benefit of integration is lower development cost, increase reliability, security and reduces the cost of the design. Integrating part can be done by embedding all the modules together and assuring their condition to work towards the goal.

\section{CONCLUSION}

The main goal of my project is to develop an autonomous robot used for detection, avoidance of obstacle and navigate through an obstacle. This project presented the use of sharp distance IR sensor for distance measurement in the development of an obstacle detection system. Automobile prototype system brings developed. It was used to sense the obstacle and avoidance of them. The implementation of an obstacle avoidance system was successfully carried out to find the minimum error by coding the algorithm in Simulink software. After obstacle detection system the signals from the IR sensor is given to motor driver through the Arduino mega 2560 microcontroller to rotate the vehicle is a predefined path. Obstacle avoidance is a very good application to be used in vehicle preventing many accident and loss of life etc.

\section{FUTURE SCOPE}

The future scope of my project is to develop an obstacle detection and avoidance system by using LIDAR sensor instead of using them different sensor. The distance measured with the help of LIDAR is more accurate as compared to other sensor and for connecting LIDAR two Arduino boards are required. The autonomous vehicles are developed in SAE second level of automation which is nothing but the partial automation in the future trying to develop the vehicle is the next level of automation. Instead of developing a system to detect the particular obstacle trying for detecting the dynamic obstacle also. And for navigation purpose, GPS and compass are using instead of the predefined path because the compass is used to navigating the car in a particular direction and calculate the correct angle and also GPS is used for navigation purpose it receives the geographical data from the satellite to calculate longitude, latitude, and speed, etc.

And for long range is 


\section{International Journal of Engineering Applied Sciences and Technology, 2019 Vol. 4, Issue 1, ISSN No. 2455-2143, Pages 120-125 \\ Published Online May 2019 in IJEAST (http://www.ijeast.com)}

\section{ACKNOWLEDGMENT}

I would like to express my deep sense of gratitude to my project head Mr. Gopal Athani from TATA Technologies Limited, Pune for providing all the facilities for completing my projects and also thanks to my guide Mrs. S. K. Apte for her valuable guidance, encouragement and kind- operation throughout the project work. Last but not the least I am very thankful to all my friends, parents and those who directly or indirectly encouraged me to take up this project.

\section{REFERENCE}

[1] Kaleemuddin S , and Bose K. (2016). Performance comparison of Infrared and Ultrasonic sensors for obstacle of different materials in vehicles/robot navigation applications, IOP Conf. Series: Materials Science and Engineering, 10.1088/1757-899X/149/1/012141.

[2] Modi.(2002). Comparison of three obstacle avoidance methods for an autonomous guided vehicle.

[3] G, Patange, S, A, \& J.(2017). Characteristics of different sensors used for Distance,Measurement.International Research Journal of Engineering and Technology (IRJET) 698-701.

[4] Varghese \& Boone. (2015). Overview of Autonomous Vehicle Sensors and Systems, Proceedings of the 2015 International Conference on Operations Excellence and Service Engineering 178.

[5] Domínguez, E, J, J, \& C.( 2011). LIDAR based Perception Solution for Autonomous Vehicles2011,11th International Conference on Intelligent Systems Design and Applications 790.

[6] Zhou, Chen, \& Xiu.(2009). A Simulation Model to Evaluate and Verify Functions of autonomous vehicle based on simulink, Springer-Verlag Berlin Heidelberg, 10.1007/9783-642-10817-4_64.

[7] Fernández, Domínguez, Fernández-Llorca, Alonso, \& sotelo.(2013). Autonomous Navigation and obstacle avoidance of a Micro-bus, International Journal of Advanced Robotic Systems, 10.5772/56125.

[8] R , Z , \& S .(2016). Obstacle-avoiding robot with IR and PIR motion sensors, IOP Conf. Series: Materials Science and Engineering, 10.1088/1757-899X/152/1/012064.

[9] Sakkhor, Zaman, \& Sadi. Autonomous Car Using Full Mapping GPS System.

[10] Tabassum, Lopa, Tarek, \& Ferdosi. (2017).Obstacle Avoiding Robot, Global Journal of Researches in Engineering.

[11] Nasucha. (2015). Development Of An Obstacle Avoiding Robot, Jurnal Sistem Komputer, 55-60.

[12] Zainal, FuadZulkifli, \& Djasmir.(2012). Development of Obstacle Avoidance Robot with Depth Obstacle and Color Signal, Journal of Applied Sciences Research,1658-1664.
[13] Chandrashekar .(2014). Characteristics Of Sonar Sensors For Short Range Measurement, International Journal of Engineering Research \& Technology,340-343. 